\title{
ADVANCED APPROACH FOR THE PUBLIC TRANSPORTATION REGULATION SYSTEM BASED ON CYBERCARS
}

\author{
Asma Melki ${ }^{1}$, Slim Hammadi ${ }^{1}$, Yves Sallez ${ }^{2}$, \\ Thierry Berger ${ }^{2}$ AND Christian TAHON ${ }^{2}$
}

\begin{abstract}
In the last decade, the authorities require the use of safe, comfortable vehicles to assure a door to door aspect with respect of environment in the urban context. In this paper, we propose an advanced approach of transport regulation where we integrate cybercars into a regulation process as an alternative in disruption cases. For that, we propose an ITS architecture including public transportation and cybercars into the same framework. We will show that collaboration between these two systems provides better results than managing them separably.
\end{abstract}

Mathematics Subject Classification. 90B06, 62C86.

\section{INTRODUCTION}

Several countries face challenges in the urban transport. The efficiency of a transport service is measured through indicators or criteria as: the immediate availability, the door to door aspect, the safety, the passenger's comfort, etc. The private vehicles satisfy these criteria and are often an effective means to realize displacements in urban zones. These displacements can involve connections between the place of residence, the offices, the shopping centers, the places of leisure activities, etc.

Received June 23, 2008. Accepted October 21, 2009.

${ }^{1}$ LAGIS École centrale de Lille, France.

${ }^{2}$ LAMIH with SP, Université de Valenciennes et du Hainaut-Combrésis, France;

christian.tahon@univ-valenciennes.fr 


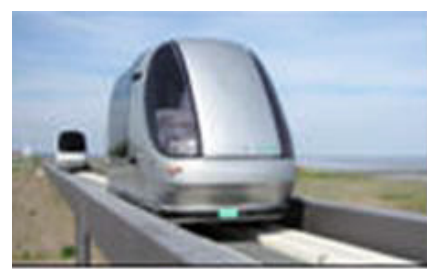

FiguRE 1. PRT.

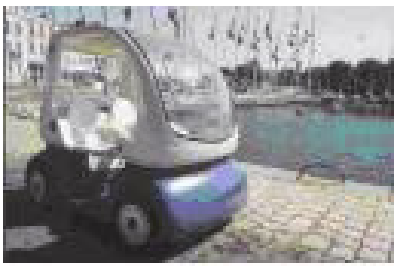

Figure 2. Cybercars.

Unfortunately, the use of the private cars causes some problems which influence the quality of life and the environment. In fact, using private cars increases traffic congestion which implies a high consumption of energy, noise, and pollution.

To cope with these problems, promising solutions have emerged following the multitude of global and european consortiums on this subject: car pooling (a car is shared by the driver and one or more passengers), car or bike sharing (rental for short periods of time), and on-demand transport with new automated vehicles types. An important advantage of the on-demand transport systems is to introduce more flexibility compared to public transportation. On the on-demand transportation system, the vehicles are synchronized with the user's need. Whereas public transportation companies supply timetables to inform and guide users, so, they have to adjust their travel with the schedules listed in the timetables.

Among these last, in [19], the author describes two main technologies: Personal Rapid Transit (small automated vehicles running on fixed guideways) and cybercars (automated vehicles able to run on any ground infrastructure without dedicated guideway). Cybercars are more suitable for a usage in city downtown, due to the flexibility of movements, the respect of the environment and its preservation of the townscape. Figures 1 and 2 illustrate the two types of automated vehicles.

The purpose of this article is first of all to situate the interaction between cybercars and Public Transportation (PT) in the Intelligent Transportation System (ITS) context, then, to enhance its contribution in the regulation of transportation system network. Sharing the same transportation network requires a coherent and efficient regulation system. The communication and data exchange have to be continuous and reciprocal. We consider a transportation system network made of a set of lines, poles of correspondence and poles of exchange. In order to restore the normal traffic, the Command and Control Center of transport (CCC) have to provide efficient strategies of exploitation. Thus, the mission of the regulators is finding the adequate strategies in a short delay by laying down some objectives as: ensuring the correspondences, minimizing the wait time of the travelers, etc. In order to support the regulators in their decision-making procedure, we propose a decision support system (DSS) made up of:

- A procedure: "The construction of feasible decisions": this procedure permits to filter the strategies giving the data about the network and the 
disturbance. The strategies which can be applied to reabsorb the disturbance.

- An evolutionary algorithm for the online regulation (OR), this strategy constitutes the most frequent strategy taken by the CCC.

- An evolutionary algorithm of the "Noria system" (explained on Sect. 4): which proposes, giving the network data, the position of cybercars, the disturbance data, etc. This algorithm provides a couple of network points indicating the starting point of the trip (in the zone of disturbance) and the point of reintegration of the travelers in the network.

- A procedure of evaluation (by a powerful aggregation tool: the Choquet integral): this procedure is integrated into the evolutionary algorithm: it consists on evaluating the set of the feasible strategies (previously elaborated) by taking into account of the criteria fixed by the companies of transportation. Indeed, when there are several possible decisions, the regulator expresses these preferences in connection with the possible actions of regulations, the scores obtained by the procedure of evaluation will give an idea on the optimal decision.

This paper is organized in six sections. In the following section, we propose through the national ITS framework, the adequacy of the two management systems in a model based on ITS requirement.

Then we detail the problem by introducing the rules of a regulation process and explaining the precisions about the advanced approach of regulation of this combined system. The third section consists on detailing the evolutionary algorithm for the online regulation strategy. In the fourth section, we explain how the Noria system proceeds to take over on the strategies implying classical transportation means.

The evaluation with Choquet Integral is the subject of the fifth section. So, we define this fuzzy integral and explain how to aggregate the strategies.

In the sixth section we give some simulation's scenarios to validate the algorithms developed previously. We conclude this paper by presenting the main ideas and the perspectives of our works. Our research is part of a project called PPF (Plan Pluri-Formation) "Cœur de Ville" which is associated with the French laboratories LAMIH ${ }^{1}$, LAGIS $^{2}$ and LGI2A ${ }^{3}$. This project aims to treat urban transport problems by using cybercars inside the city.

\footnotetext{
${ }^{1}$ LAMIH: Le Laboratoire d'Automatique, de Mécanique, et d'Informatique industrielles et Humaines.

${ }^{2}$ LAGIS: Le Laboratoire d'Automatique, Génie Informatique et Signal.

${ }^{3}$ LG2IA: Laboratoire de Génie Informatique et d'Automatique de l'Artois.
} 


\section{INTELLIGENT TRANSPORTATION SYSTEM AND CYBERCARS}

\subsection{INTELLIGENT TRANSPORTATION SYSTEM}

Various definitions of Intelligent Transportation Systems (ITS) have been put forward, we present, in this context, the definition proposed by ITS community of Canada: "The application of advanced and emerging technologies (computers, sensors, control, communications, and electronic devices) in transportation to save lives, time, money, energy and the environment"4. The intention of the ITS community is to cover all modes (ground transportation such as private automobiles, commercial vehicles, and public transportation, and also rail, marine, and air modes). The dynamic character of ITS allows to cover the vehicle, the infrastructure and the driver or user, interacting together dynamically.

\subsection{Cybercars}

The following definition is given by Parent in [17] "Cybercars are road vehicles with fully automated driving capabilities. A fleet of such vehicles forms a transportation system, for passengers or goods, on a network of roads with on-demand and door-to-door capability". Since 2001, several European projects: Cybercars ${ }^{5}$, Cybermove $^{6}$, NetMobil ${ }^{7}$, CityMobil ${ }^{8}$ have check how on-demand automated vehicles might improve mobility while enhancing the attainability [18]. The main advantages of cybercars exhibited by the cited before projects are:

- Availability: allowing users to find a vehicle at different times of the day.

- Usage friendly: allowing disabled or elderly persons to travel more easily in the city.

- Redeployment: the cybercars move easily from one location to another, and can be stored to parking area, leaving valuable urban space free.

- Reduction of congestion and better air quality and energy conservation.

- Environmental cost.

For cybercars, as for any means of transport, we have to take into account many aspects of quality of service (QoS) like the cost, the safety, the maintenance and travel demands [13]. To incorporate these aspects, and not to rediscover solutions to usual needs, we situated the problem in the ACTIF model ${ }^{9}$.

In the following section, we present the ACTIF model and the interactions between Cybercars and mass transit modes.

\footnotetext{
$4_{\text {www . itscanada. com }}$

${ }^{5}$ http: //www . cybercars .org

${ }^{6}$ http://www. cybermove.org

${ }^{7}$ http://www. netmobil.org

${ }^{8}$ http://www.citymobil-projetc.eu

9 http://www.actif-its.org
} 


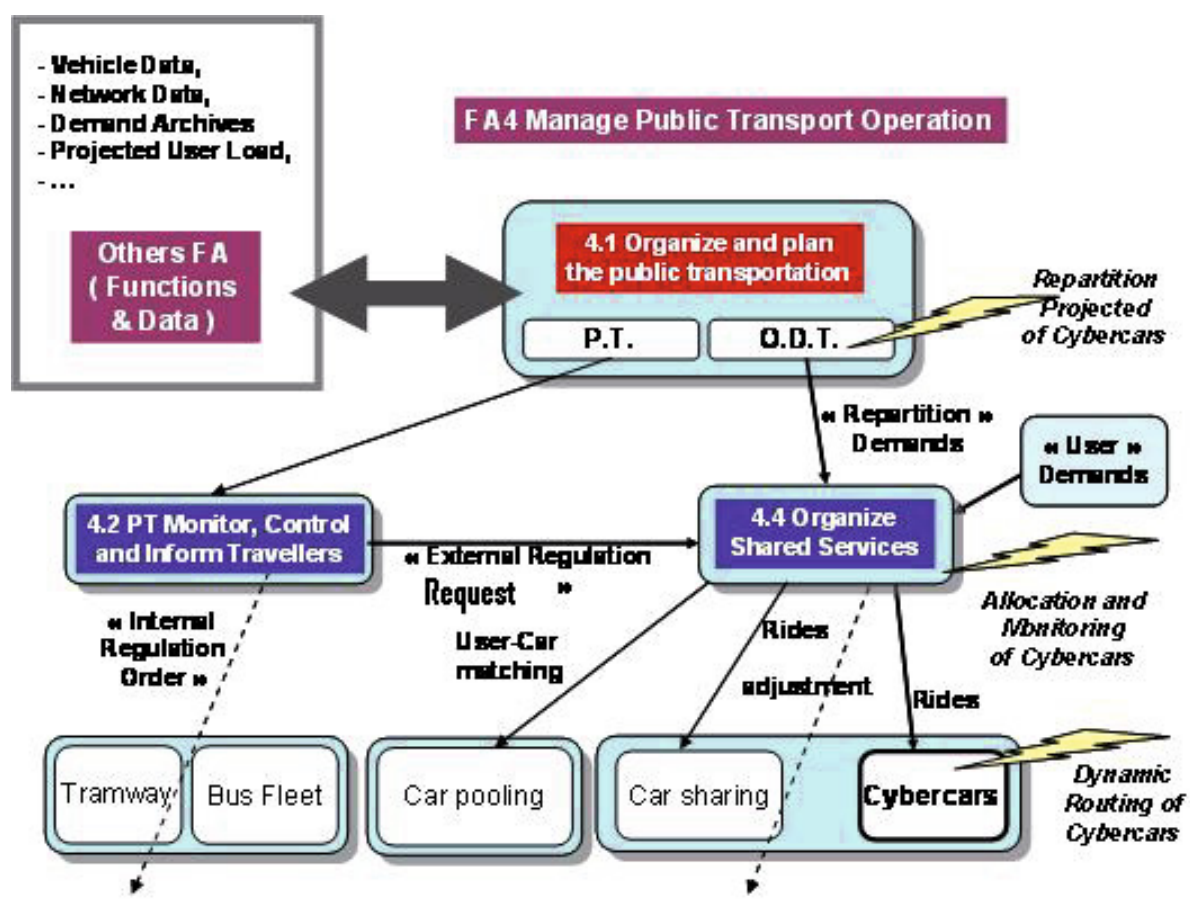

FiguRE 3. ACTIF model integrating Cybercars.

\subsection{ACTIF MODEL}

ACTIF (Aide à la Conception des systèmes de Transports Interopérables en France) is the French ITS Framework Architecture related on the FRAME (Framework Architecture Made for Europe $)^{10}$. ACTIF is used here as a generic model of an infrastructure of different means of transport; complete enough to allow the positioning of the cybercars system (and Public Transportation) with respect to the aforementioned aspects. ACTIF is used because it is the current ITS model in France, derived from KAREN (European ITS model). ACTIF is organized in several functional domains. The functional domain "Manage public transport operation" is particularly adapted to introduce the cybercars system. This domain is divided into five sub-domains from FA4.1 to FA4.5. Figure 3 summarizes the sub-domains which make reference to the interconnection between the PT system and the shared services.

The sub-domains envolving the interaction between public transportation and cybercars are:

\footnotetext{
${ }^{10}$ http : //www . its-world
} 
- FA4.1. Organize and plan the public transportation: manages the planning and organization of the production of services and the management and maintenance of resources.

- FA4.2. Supervise, regulate and inform passengers: monitors and controls traditional Public Transport (e.g. bus, tramway). Furthermore, this subdomain manages information aid system for travelers.

- FA4.4. Organize shared services: manages the planning and organization of shared services in general: public transport, such as on-demand services or the management of shared vehicles, but also private initiatives such as car pooling.

The cybercars system is therefore included in the sub-domain FA4.4. This subdomain allows to encompass the outline of the cybercars system. In fact, cybercars system need some additions in 4.1 and 4.4. Therefore we propose to break down sub-domain 4.1 into two parts: one part manages the traditional Public Transports (PT) and the other part manages the On Demand Transport. In ODT, by taking into account of the projected demands, 4.1 calculates the "Repartition Projected of Cybercars". The repartition is the positioning of the free cybercars in space. This for responding quickly to the users demands, in minimizing the users waiting of free cybercars moving towards these users. The results are "repartition demands".

Specifically for the cybercars, F.A 4.4 calculates the allocation of the free cybercars to users by taking into account the "users demands". The result are the cybercars rides. A ride is a moving order, from one localization to one destination, with one specific cybercar for one user or one group of some users. A ride (in this case it is a repartition ride) can also be only the moving of a free cybercars to a destination for waiting a potential user. FA4.4 includes the monitoring of all cybercars on the rides. If needed, an adjustment can apply to the cybercars, for example to modify the moving of free cybercar, or adjusting the cybercars speed. The effective moving of the cybercars, by taking into account in realtime the traffic perturbations, can be based, for example, on a distributed dynamic routing approach [14,24]. Another aspect concerns interaction between cybercars and humans is detailed in [21].

By analyzing the functional domain quoted above, one can observe that data flows related to these domains make the connection between certain functions where public transport and the shared services are found.

Indeed, in ACTIF, it is indicated that sharing vehicles are the voluntary or paying services:

- vehicles shared without driver placed at the disposal of the public by public and deprived organizations;

- carsharing;

- individual or collective taxis;

- services of ambulances;

- etc.

To make an adequacy between supply and demand, it is carried out to bring together the public transportation services and the shared services. 


\subsection{INTERACTION BETWEEN PUBLIC TRANSPORTATION AND CYBERACARS}

We thus chose a model in Figure 3 which gathers these two actors of transport (e.g: PT and Cybercars) and which takes care to make them collaborate in order to ensure a sustainable mobility. The model suggested connects functional subdomain of the ACTIF model. We can distinguish the data flows which interact between them. In this model, we distinguish two types of regulation requests:

(1) Internal Regulation Request or Order (IRR).

The decision actions which imply public vehicles (tram, bus, etc.) are considered as Internal Requests of Regulations (IRR). As an example of these decisions, we cite: the suppression or the creation of a trip, the resorption, the deviation, etc.). The actions relating to the bus and tram are detailed later on (Sects. 2.2 and 2.3).

Studies showed that the most critical disturbances are those where the tram undergoes an incident. The standstill of the oars of the tramway as an emergency procedure involves heavy operations to restore the command because the injection of new oars coming from the deposit is often not the effective solution. In addition, when disturbances include different lines, the horizon of regulation is widened considerably. In this case, the actions of delay/advance which one applies for the buses will take more time to restore the normal operation of services of transport according to the length of the affected line. These reasons lead to conclude that it is judicious to bring new measures of regulation to the urban transportation systems.

(2) External Regulation Request (ERR).

On the exploitation of public transport level, we distinguish a data flow "action of regulation" which could be introduced like an input for the collection of the demand for shared services. The interaction between public transport and the shared services will be active on this side. Indeed, the organization of the shared services is done in three functions:

- collection of supply and demand;

- organization of the sharing of the vehicles;

- rapprochement between supply/demand.

The ERR is introduced on the level of collecting the supply and demand of the shared services. The aim of sending this request is to elaborate a strategy of exploitation based on integrating the cybercars to solve a critical disturbance. Nevertheless, the fleet of cybercars can undergo disturbances. Some approaches of resolution of the routing problem based on bio-inspired algorithms are mentioned in [20].

\section{Regulation APPROACH}

In the regulation process, the information indicating any modification on the network behaviour is transfered from the Information Support System (ISS), this 


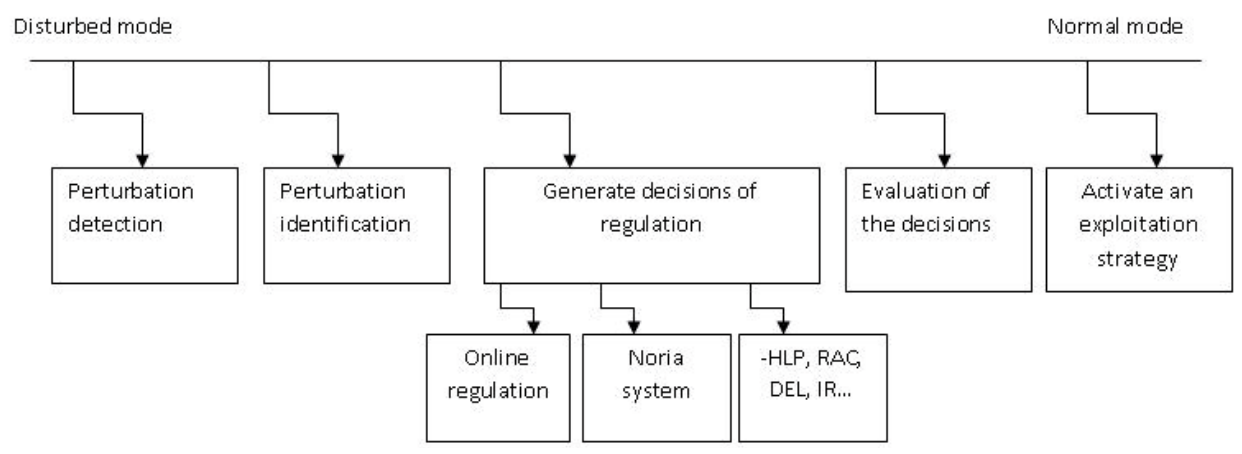

FIGURE 4. Advanced approach of regulation.

information is transmitted as an alert to the Exploitation Support System (ESS). Through this routing of information, the process of regulation is activated, it consists on applying strategies to restore the normal operating mode from a disrupted mode (travelers could refer again to the theorical schedules elaborated at the planning phase of the transportation management system) [15]. It requires a polyvalent acknowledgements and can be studied in different ways. Several researchers focused on the conception and evaluation of a traveler Information system $[6,7,11]$. Other works have enhanced decision-making process. The goal is to reproduce the behavior of the regulator to give birth to a computerized decision system [16].

In a real time context, the regulators have to face several disruptions occuring at the same time on different places of the network. Even if the Decision Support System (DSS) is able to readjust a traffic affected by two disturbances, its performance can be improved for faster action.

Furthermore, some decision classes increase drastically the operation costs because they require the use of human or material resources. It would be possible to combine the advantages of cybercars to the performance of the DSS, to improve the transport system of the future. We will consider the cybercars as the heart of a "Noria process". The "Noria" in transportation field is a process of supply chain inspired from the hydraulic irrigation system.

By analogy, this system joins two relay points, avoiding long distances journey made by the initial transportation mode by switching this transportation task to another mode more efficient in court distances or more suitable in urban environment. In Figure 4, we illustrate our advanced approach of regulation which aims to return to the normal mode of exploitation starting from the disturbed mode.

Integrating cybercars into a regulation system is, in practice, traduced by adding a new decision class to the register of bus and tramway decision classes. The decision class related to cybercars is the "Noria System". When a critical disturbance is detected, the regulator explores other strategies by sending to the private sharing transportation provider an ERR (Sect. 1.4). Thus, a "Noria process" proposes to the users to continue their trip by modifying the initial course and to use the cybercars to help them to avoid the disturbance zone. 
We worked on the hypothesis that an extra decision strategy will be included in the regulation process: when different disruptions are located on the network, a global scan is made to situate the priorities of the intervention and the ERR is sent to the cybercar transport provider to activate a "Noria process". When a disturbance is detected by the ISS, this information is transmitted to the ESS. An analyze of the perturbation is made to reduce the decision horizon. This procedure will increase the reactivity of the regulator vis-a-vis a problem.

\subsection{Construction of the Decisions}

The construction of the feasible decisions is carried out by an inductive reasoning. Starting from a base of knowledge and a base of rules, an inference engine generates the feasible solutions.

In the following, we detail the basic elements of the inductive reasoning: the base of knowledge is composed of:

- The frequency of the line.

- The line and the direction.

- The value of the delay of the disturbed vehicle.

- The hour of the day.

- If the disturbance causes a specific blocking or a general blocking.

- Visibility or not of the vehicle following the disturbed vehicle.

- Weather conditions (good weather/bad weather).

- Schedule of the disturbance.

- Estimated duration of the action.

- The number of vehicle of reserve and available drivers.

There are also static data included in the knowledge base such as: the maximum capacity, the number of stations of the line, the beat, etc. The possible decisions generated by the inference engine are divided into operations or manœuvers applied to the bus network and the tramway network.

\subsection{BUS MANEUVERS}

The regulator indicates some operations to the bus conductor. These operations consists on modifying partially the itinerary or the course.

(1) Deviation.

(2) Online express.

(3) Online half-turn.

(4) The half-turn with the driver and vehicle exchange.

(5) Overtaking and service in descent only.

(6) Departure delayed in the terminus.

(7) The driver and vehicle exchange.

(8) The shortcut (SC).

(9) Injection of a reserve and available (IR).

(10) Online regulation (OR).

(11) Absorption in terminus (AET). 


\subsection{Tramway maneuvers}

The Tramway system is managed to adapt with the traffic conditions:

(1) Exchange at the end of the turn.

(2) Deviation.

(3) Towing/pushing.

(4) Limited speed.

(5) Fold of reserve.

(6) Restoration of service.

(7) Wait agent.

The Online Regulation (OR) is appreciated by the regulators considering its facility of execution. Nevertheless, in order to generate a regulated timetable which will carry out at the end of a certain time to the theoretical timetable, an intervention on the starting and arrival schedules of the vehicles is necessary, while optimizing the time of total course and by respecting the constraints of the network. For these reasons, it would be more adequate to generate the decision of online regulation by a multi-objective optimization algorithm. We adopt a genetic algorithm for its capacity to resolve NP-problems.

\section{ThE ONLINE REGULATION}

\subsection{DeCision VARIABles}

To codify the system solutions, we use a variable that reflects the passage of a vehicle when it passes by the station where the disturbance was detected. Indeed, the online regulation does not take into account the moments before disturbance.

The codification must represent the vehicle that undergone the disturbance, the station where we detect the dysfunction and the action to be initiated.

So, we used a matrix coding where the lines correspond to the stations and the columns to the vehicles. The content of each element of the matrix corresponds to the instruction to carry out. This coding was elaborated in [1]. A possible instruction to give to the conductor can be a request to delay the vehicle thus to stop for one period given to the station or to advance more quickly the vehicle what corresponds to an operation of acceleration or anything to change with the behavior of the conductor and thus nothing to make. In a real case, it is almost excluded to ask a driver to accelerate. The regulation on line thus consists in maintaining a certain form of regularity between the vehicles all while delaying them.

\subsection{INITIALIZATION OF THE POPULATION}

The population of the solutions must be on the regulation zone, thus it is necessary to limit the generation at the lower and upper boundaries of the zone. If the primary decisions are missing, we begin with a random generation of the solutions. 
TABLE 1. Selection operator.

\begin{tabular}{|c|}
\hline Selection operator \\
Step 0 : initialization \\
Step 1 : for each individual $i$ to PopSize \\
calculate $e=N \cdot p_{i}$ \\
Select $i E\left(e_{i}\right)$ times \\
Step 2 : calculate the rest $r_{i}=e_{i}-E\left(e_{i}\right)$ \\
select the individual $i$ at most, as many time as $E(i)$ with the probability of $r_{i}$
\end{tabular}

$E(i)$ is the whole part of the number $i$.

\subsection{Evaluation of the population}

The criteria which can be affected by online regulation are the regularity and the correspondence criteria. The commercial kilometers and the quality of service are not, considering the distance covered still the same one and the parameters of the measurement of the quality of service are not modified. The evaluation of its criteria is carried out by the Choquet Integral. This stage will provide us the optimal individual as well as the optimal timetable

\subsection{The SELECTION OPERATOR}

The operator of selection used is the selection by the improved wheel of Goldberg. This type of selection allows a better diversity of the individuates.

\subsection{The CONTROLLED CROSSOVER OPERATOR}

The matrix form of the chromosome gives a priori the choice to carry out the operation of crossing on the lines of the stations or those of the vehicles. The principle of a uniform crossover is to establish a binary mask generated by chance same length as the genotypes. If with the $n$th position, there is 0 , we preserves the symbols if not we exchange the elements. This operator is controlled to limit the delays

\subsection{The CONTROLLED MUTATION OPERATOR}

To diversify the solutions, we carry out a mutation with a probability $p_{\text {mut }}$. As for the crossover operator, the mutation operator is controlled in order to avoid the delays.

\subsection{The ONLINE REGULATION ALGORITHM}

We present on the following table the different steps of the online regulation algorithm. 
TABLE 2. Online regulation algorithm.

\begin{tabular}{|c|}
\hline Online regulation algorithm \\
Step 0 : initialization \\
initialize P \\
evaluate individuals with Choquet Integral \\
while (notmax-generation) do \\
begin \\
Step 1: selection \\
Step 2: crossover \\
Select the best individual of 1 at generation to construct $\mathrm{P}^{*}$ \\
Select randomly two parents of $\mathrm{P}^{*}$ \\
crossover, pcross \\
put children and non crossed parents in P \\
Step 3: mutation \\
mutation of P individuals,pmut \\
Step 4: evaluate P \\
for each P individual, calculate the regulated timetable, Charge, cost \\
Save the best individuals \\
Step 5 : comparaison \\
next generation \\
repeat from Step 1
\end{tabular}

\section{Noria System ALGORIthm}

As for the online regulation, the Noria system is a decision which is easy to set up considering that it does not require modifications of the journeys or the deployment of human resources. The management system of the cybercars can be independent of the management system of public transportation. When the disturbance is a breakdown or an unspecified failure and the delay is estimated at more of the tolerable $12 \mathrm{~min}$ by the transportation service provider. In addition, the travelers getting out of a broken down bus will have the impression that they have less waited by knowing that they will be transported directly by another mean of transportation. Whereas, the travelers, knowing that they have to wait the same transportation mean which underwent the incident, will express their discontent. Indeed, the impact of the traffic situation influences considerably the quality of service offered to the user. Starting from a theoretical point of view, the situation is more complex since the noria system effectiveness depends closely of the (origin/destination) stations corresponding to the removal and the re-injection points on the network. 


\subsection{CYBERCARS CONSTRAINTS}

Thanks to the several evaluation reports of Cybercars, we can delimit the following constraints:

- Each cybercar has only one starting point and only one point of destination.

- Each cybercar has a maximum capacity.

- The cybercars make short distances, we thus draw aside the courses which are higher than $5 \mathrm{~km}$.

- The speed of these vehicles is limited at around $30 \mathrm{~km} / \mathrm{h}$ but this is quite sufficient in many urban environments.

\subsection{NORIA PROCESS}

Let $t_{\text {dis }}$ the time where delay is treated, $t_{\text {arm }}$ the arrival time of the disrupted mode at station $S_{\text {up }}$ and $t_{\text {arc }}$ the arrival time of the cybercars to the node where travelers will be picked up

At $t_{\mathrm{dis}}$, the number of passengers in transfer is a percentage of the travelers transferred from the previous mode. Thus, we calculate the total time of the journey between $S_{\text {up }}$ and the station where the travelers will be injected $S_{\text {down+1 }}$; several works elaborated methods to compute the waiting time of passengers in transit. We cited in particular the following works: in [2], a mathematical model is developed to analyze the passenger waiting time of an intercity transit station with feeder bus services. This influences the reliability of feeder bus services. Shen [22] shows that holding strategies combined with short-turning strategies reduced the mean passenger waiting time in case of disruptions. In [12], the author computes the wait time considering the interval time between the passage of successive vehicles and the number of travelers in the station, where the arrival of passengers is assimilated to a Poisson processus.

In our problem, two cases could be distinguished :

- In the ERR, public transportation provider informs that all passengers must be evacuated and transferred to the next reinsertion node and the sharing service provider send the number of vehicles needs in the entering point of the "Noria process". Let's consider: $t_{\text {arp }}$ : the arrival of passengers to the pick up point. $t_{\text {arc }}$ : the departure of the vehicle. In the case that passenger's flow will be totally absorbed, the waiting time will be calculated as the time spent between $t_{\text {arp }}$ and $t_{\text {arc }} \operatorname{Tot} T=t_{\text {arc }}-t_{\text {arp }}$

- Public transportation provider consider that the disruption has not a higher priority, so passengers will be progressively picked up each time that a vehicle is send by the sharing service provider. This time increases proportionally with the incoming travelers from the deficient mode. $\operatorname{Tot} T=\int_{0}^{\Delta(t)}\left(\operatorname{ratio}_{\mathrm{psg}}(\Delta(t)-t)\right.$ 
A genetic algorithm is used to provide the reinsertion point giving the station where disturbance is detected and the data about bus and tramway lines. The evaluation of the actions of decisions (e.g: OR, Noria process, Deviation, etc.) gives to the regulators an illustration of the impact of these decisions if they are applied. The simulation of the scenarios will avoid the possible errors of judgement of traffic situation. The aggregation of the criteria, according to the possible decisions, is an important step of the decision-making process. The Choquet integral permits to aggregate criteria and to take into account the interaction between them. On the next section, we detail the evaluation process.

\section{Evaluation with Choquet integral}

\subsection{MULTICRITERIA DECISION MAKING}

On multicriteria decision making problems, several aggregation methods are used, we cite mainly the weighted arithmetic operator (OWA) and the mean operator [8]. In numerous problems, the criteria considered interact but, unfortunately, those tools do not permit to express this interaction and to interpret them correctly. Thanks to their characteristics and mathematical properties, the fuzzy integral are used in order to overcome these weak points. The are two fuzzy integrals [25] that interest the researchers on decision-making problems: The Sugeno Integral [23] and the Choquet integral [3]. A characterization of fuzzy integral is the additive measure, for more details we refer the readers to [9]. This measure could be sub-additive for some subsets of the criteria and super-additive for another subset of criteria. The choice of Choquet integral in our evaluation process is due to the fact that $\lambda-$ Sugeno measures are either sub or super additive. An academic example is presented on [10] to show the Choquet integral performances.

\subsection{Choquet integral}

Definition. Let $\mu$ be a fuzzy measure on $\mathrm{X}$.

The Choquet integral of a function $f: \mathrm{X} \longrightarrow[0,1]$ with respect to $\mu$ is defined by:

$$
\mathcal{C}_{\mu}\left(f \left(x_{(1)}, \ldots, f\left(x_{(n)}\right):=\sum_{i=1}^{n}\left(f\left(x_{(i)}\right)-f\left(x_{(i-1)}\right) \mu\left(A_{(i)}\right)\right.\right.\right.
$$

where ${ }_{(i)}$ indicates that the indices have been permuted so that:

$$
0 \leq f\left(x_{1}\right) \leq \ldots \leq f\left(x_{n}\right)
$$

\subsection{The Choquet integral For 2-Additive measures}

By applying some modifications to the basic form of Choquet integral using the Shapley index. Grabisch [9] presented the expression of the Choquet integral to be applied in case of 2-additive measures as a combination of a conjunctive, 
disjunctive and an additive part which express respectively positive interaction indices, negative interaction indices and the Shapely Value [5]:

$$
\begin{aligned}
\mathcal{C}_{\mu}\left(t\left(x_{1}\right), \ldots, t\left(x_{n)}\right)=\sum_{I_{i j}>0}\left(t_{i} \wedge\right.\right. & \left.t_{j}\right) I_{i j} \\
& +\sum_{I_{i j}<0}\left(t_{i} \vee t_{j}\right)\left|I_{i j}\right|+\sum_{i=1}^{n} t_{i}\left(v_{i}-\frac{1}{2} \sum_{j \neq i}\left|I_{i j}\right|\right) .
\end{aligned}
$$

This expression is practical in multicriteria decision systems.

The appreciations of the decision maker are traduced through a set of criteria. The evaluation of these criteria aims to preserve a balance between satisfying travelers by providing an acceptable level of quality of service as well as ensuring the profit to the transportation company. On the following subsection we enumerate the criteria validated with a regional french transportation provider and the new criteria respecting the urban environment context of "Noria process".

\subsection{CRITERIA OF THE PUBLIC TRANSPORTATION SYSTEM}

The collaboration with the transportation service provider

- Correspondence $(\mathrm{CR})$ : the criterion of correspondence is associated the duration of the transfers between the vehicles to a pole of exchange.

- Punctuality (PT): is associated the respect of the schedules of passage and thus of the durations of course. it is a question of optimizing the total duration of the courses aboard various vehicles, according to the number of travelers.

- Commercial kilometers (KM): it is a question of minimizing the difference between the theoretical and the real distances traversed by the various vehicles, this criterion is very significant if the transportation company decides to change the itinerary of a vehicle or to reconfigure the network.

- Regularity (RT): this criterion corresponds to the regularity of the time intervals which separate the successive passages from the vehicles at the same station. It relates to the minimization of waiting time of the travelers to the stations of the network. The waiting time considered in this criterion concerns only the travelers who came "on foot" to the stops.

- The Quality Service (QS): the measurement of the quality of service can be different from a company of transportation to another but they all agree on the importance of this criterion. We chose in assent with our industrial partner Transvilles to measure the quality of service by the number of not served stations, the number of exchanges of vehicles and conductors and the number of transshipments. 


\subsection{CRITERIA OF CYBERCARS SYSTEM}

The characteristics of a cybercar are different from those of the traditional means of transportation. The authorities of transport particularly support the means known as "clean" compared to the other modes operating in urban cities, and permit the development of the shared service companies. Based on the evaluation reports of the Cybercar and CyberMove projects and the coordination with the various teams of the project "coeur de ville", we elaborated some criteria in order to evaluate the noria system. The cybercars has specific criteria which could be summarized on: commercial kilometers, waiting time of customers, environmental cost, quality of service.

(1) Commercial kilometers (KM): this criterion represents the distance crossed in kilometers that the sharing service provider has to assure.

(2) Waiting time of customers (RT): the objective of the "Noria process" is to reduce the waiting time of travelers between the moment where a disruption has occurred and the pick up.

(3) Environmental cost (CE): this is an important criterion as it reflects the awareness of transportation authorities to preserve the environment. Notice that, even if cybercars energy consumption is reduced behind other public transportation, they still use either hybrid energy or electric energy and needs to be rationalized. Several studies focuses on determining mathematical formula traducing a causal relations between the consumption, the use of means of transportation and the effect on the environment [4].

(4) Quality of service (QS): the security of travelers and in particular disabled persons is a priority factor. Thus, in "Noria System", the sharing service provider attribute a weight to the pick up charge of this category

In simulation, we consider the following symmetric matrix which expresses the interaction with criteria. The diagonal represents the weights or utility function attributed to each criterion. $I_{i j}=\left(\begin{array}{lllll}w_{1} & I_{12} & I_{13} & I_{14} & I_{15} \\ I_{21} & w_{2} & I_{23} & I_{24} & I_{25} \\ I_{13} & I_{23} & w_{3} & I_{34} & I_{35} \\ I_{14} & I_{24} & I_{34} & w_{4} & I_{45} \\ I_{15} & I_{25} & I_{35} & I_{45} & w_{5}\end{array}\right)$.

This matrix permits to calculate, for every generated decision class, a global score. It also permits an aggregation of the different criteria cited on Sections 5.4 and 5.5. The diagonal expresses the individual score for each criteria.

\section{Scenarios and Results}

For simulation purposes, the decisions related to bus system are adopted for tramway system. But, one cannot apply all the possible decisions of the bus to the tram, considering this last is relatively related to its infrastructure.

So we selected the decisions which can apply jointly for the two systems:

- online express (OEX); 
- departure delayed in terminus (DDT);

- online regulation $(\mathrm{OR})$;

- absorption in terminus (AIT).

In this section, we present different scenarios of disturbances through simulations elaborated on offline mode on the transportation network of Valenciennes (City in the north of France).

\subsection{SCEnARIO 1: ONE Disturbance}

Let's consider a "Delay of Tramway at Mairie Anzin station at 6:01" as an incident

The delay occurred on an off-peak hour. To simulate the advanced approach of regulation we give the different steps made of our First of all, the procedure of construction of decision provides the feasible solutions

- online express (OEX);

- departure delayed in terminus (DDT);

- online regulation (OR);

- absorption in terminus (AIT).

Then we integrate those feasible solution into the the evaluation process based on Choquet Integral.

We obtain a global score of 0.67 associated to the online regulation (OR), the worst score is 0.13 attributed to the absorption in terminus (AIT).

\subsection{SCEnARIO 2: DisturbanCES ON PEAK-HOUR}

At 11:42, the Exploitation Support System transmits two simultaneous disturbances to the DSS: a delay of 5 min of a bus detected at "Postillon station" (line 15) and, at the same time, a breakdown of bus at "Albert I station" (line 63) is detected.

In the following, we treat the first incident.

\subsubsection{First incident}

The delay observed on Postillon station occurred at the end of the line which has as a destination "Crespin". The algorithm of construction of the decisions proposes: absorption in terminus (AIT) and Noria system (NS).

We introduce the data of the first incident into the "Noria system" algorithm. The results given are: $\left(S_{28}^{15}, S_{23}^{16}\right)$. This couple of stations indicates the station where passengers are picked up and the second station indicates to station where passengers are injected into the transportation network.

Evaluation of the DSS. We evaluate the decisions resulting from the construction decision algorithm specific to DSS.

Evaluation of the Noria System. We evaluate the Noria system intervention.

We conclude that using a Noria system is preconized in this case while the Absorption in terminus gives a lower efficiency. 
TABLE 3. Evaluation with DSS.

\begin{tabular}{|l|c|c|c|c|c|c|}
\hline & \multicolumn{5}{|c|}{ Criteria } \\
\hline Decision classes & AT & TT & RT & KM & QS & Global score \\
\hline AIT & 0.27 & 1 & 0 & 0.75 & 0.3 & 0.44 \\
\hline
\end{tabular}

TABLE 4. Evaluation of Noria system.

\begin{tabular}{|l|c|c|c|c|c|}
\hline & \multicolumn{5}{|c|}{ Criteria } \\
\hline Decision classes & CE & RT & KM & QS & Global score \\
\hline NS & 1 & 0.3 & 0.75 & 1 & 0.45 \\
\hline
\end{tabular}

TABLE 5. Evaluation of the DSS.

\begin{tabular}{|l|c|c|c|c|c|c|}
\hline & \multicolumn{5}{|c|}{ Criteria } \\
\hline Decision classes & AT & TT & RT & KM & QS & Global score \\
\hline AIT & 0.67 & 1 & 0 & 1 & 0.1 & 0.45 \\
\hline
\end{tabular}

TABLE 6. Evaluation of the Noria system.

\begin{tabular}{|l|c|c|c|c|c|}
\hline & \multicolumn{5}{|c|}{ Criteria } \\
\hline Decision classes & CE & RT & KM & QS & Global score \\
\hline NS & 1 & 0.27 & 1 & 1 & 0.70 \\
\hline
\end{tabular}

\subsubsection{Second incident}

Line 63 was affected by a disturbance detected on the station "Albert I" in the direction of "Maing Rucart". This station is at the beginning of the line. The algorithm of construction of the decisions proposes the following feasible strategies:

- Absorption in terminus (AIT).

- Injection of Reserve (IR).

The Noria system algorithm provides the solution: $\left(S_{5}^{63}, S_{5}^{62}\right)$. When we observe the displacement plan, it is shown that $\left(S_{5}^{62}==S_{7}^{63}\right)$.

Evaluation of the DSS. We evaluate the criteria of the multimodal transportation system according to the AIT manœuvers.

Evaluation of the Noria system. We conclude that in this case the "Noria system" is recommanded to resorb this incident.

\subsection{SCEnario 3}

Now, we consider a scenario more complicated, Let's consider a disruption that affect 4 lines: 11 way 1 , line 15 way 1 , line 16 way 2 and the tram way 2 .

We suppose that at 10:50, the tram has a mechanical problem causing a delay of 10 minutes, the arrival time will be at 11:06 inspite of 10:56. It has 3 corresponding 
TABLE 7. Evaluation of the DSS.

\begin{tabular}{|l|c|c|c|c|c|c|}
\hline & \multicolumn{6}{|c|}{ Criteria } \\
\hline Decision classes & AT & TT & RT & KM & QS & Global score \\
\hline SC & 0.51 & 1 & 0 & 0.7 & 0 & 0.42 \\
\hline OEX & 0.67 & 1 & 0 & 1 & 0.1 & 0.45 \\
\hline OR & 0.32 & 0.83 & 0.17 & 1 & 1 & 0.62 \\
\hline
\end{tabular}

TABLE 8. Evaluation of Noria system.

\begin{tabular}{|l|c|c|c|c|c|}
\hline & \multicolumn{5}{|c|}{ Criteria } \\
\hline Decision class & CE & RT & KM & QS & Global score \\
\hline NS & 0.52 & 1 & 0 & 0.7 & 0.72 \\
\hline
\end{tabular}

nodes. The first at 11:05 with bus 14 of line 11. The second at 11:06 with bus 9 of line 16, the last one with bus 15 on line 15 at 11:06.

The algorithm of construction of decisions provides the following possible regulation procedures:

- Shortcut (SC).

- Online express (OEX).

- Online regulation $(\mathrm{OR})$.

\section{Evaluation of the DSS.}

In this example, the best score refers to the online regulation (OR) (Tab. 7), thus the regulator has to apply an operation strategy: delaying some upstream and downstream vehicles of a disrupted one in order to avoid excess load and to help it not to increase its delay, and to restore the interval regularity.

\section{Evaluation of the Noria system.}

We execute the Noria system when a disruption is detected and we apply this process refereing to the regulation zone.thus and for the same circumstances than the classical decision classes, thus we intervene. Results are shown by the following table. In this case and due to the disturbance position, the Noria system procedure could be applied and gives better results than an OR procedure.

\subsection{Conclusion}

Simulations gives us an interesting results as it permits to prove that integrating a Noria System accentuate the global score. These results depends, in fact, on the disruption position. A perturbation that occurs near from bus depot gives better results if a classical decision class of the DSS is adopted instead of the use of Noria system procedure. 


\section{Conclusion And PeRspectives}

In this paper, we proposed an intelligent mobility system that combines regular modes and a new concept of vehicles based on soft computing and new technologies: the cybercars. This combination offers to passengers a better way to move while respecting the environment, increasing security and benefit from the different alternatives proposed by PT and cybercars service providers. After presenting a normalized architecture of this system, we detailed the different constraints, criteria that integrate the global regulation process. At least, A decision support system based on Choquet integral for the evaluation of this decision classes was tested through real scenarios that could happen at any moment of the journey and could be embarrassing for the travelers if not resolved in an efficient manner. This work introduces the different possibilities to integrate cybercars in a urban context, not only to realize displacements but also to participate to the regulation process. On future works, we study the effect of cybercars system dispatching on regulation process.

\section{REFERENCES}

[1] M. Aloulou, Application des algorithmes génétiques à la régulation du trafic des bus. Master thesis, Université des Sciences et Technologies de Lille (1999).

[2] S. Chang and H. Chun-Lin, Modeling passenger waiting time for intermodal transit stations. Annual Meeting of the Transportation Research Board 1 (2001) 69-75.

[3] G. Choquet, Theory of capacities 5 (1953) 131-295.

[4] J. Dearien and M. Plum, The capital, energy, and time economics of an automated, ondemand transportation system. IEEE AES Systems Magazine (1993).

[5] D. Denneberg and M. Grabish, Shapley value and interaction index, in Information Sciences, (1999) 149-170.

[6] H. Ezzedine, T. Bonte, C. Kolski and C. Tahon, Integration of traffic management and traveller information systems: basic principles and case study in intermodal transport system management. IJCCC 3 (2008) 281-294.

[7] H. Ezzedine, C. Kolski and A. Peninou, Agent oriented design of human- computer interface. Application to supervision of an urban transport network 18 (2005) 255-270.

[8] M. Grabisch, Fuzzy integral in multicriteria decision making, in Fuzzy Sets and Systems 69 (1995) 279-298.

[9] M. Grabisch, Pattern Recogn. Lett. 17 (1996) 567-575.

[10] M. Grabisch and M. Roubens, Fuzzy Measures and Integrals - Theory and Applications, in Application of the Choquet Integral in Multicriteria Decision Making, Physica Verlag (2000) 415-434.

[11] S. Lepreux, M.-A. Hariri, J. Rouillard, D. Tabary, J.-C. Tarby and C. Kolski, HumanComputer Interaction. Springer-Verlag (2007) 134-143.

[12] Y. Li, Real-time scheduling on a transit bus route: A 0-1 stochastic programming model. Technical report, Centre de Recherche sur le Transport, Université de Monréal (1991).

[13] M. Mcdonald and T. Voge, Analysis of potentials and limitations of cybernetic transport systems. Technical report.

[14] A. Melki, F. Dimutruscu, Y. Sallez, T. Berger and S. Hammadi, Interaction des transports publics et des cybercars dans un contexte urbain. IEEE LT (2007).

[15] A. Melki and S. Hammadi, Hierarchic decision support system approach with evaluation of decision classes of a traffic network transportation. IEEE SMC (2006). 
[16] M. Ouldsidi, Contribution à l'amélioration des systèmes d'aide à la décision pour la régulation du trafic dans les réseaux de transport collectif. Ph.D. thesis, École centrale de Lille (2006).

[17] M. Parent, Cybercars: A solution for urban transport, in CODATU Conference (2004).

[18] M. Parent and G. Gallais, Cybercars: Review of first projects. Ninth International Conference on Automated People Movers.

[19] M. Parent and G. Gallais, Intelligent transportation in cities with cts. ITS World Congress.

[20] Y. Sallez, T. Berger and D. Trentesaux, A stigmergic approach for dynamic routing of active products in FMS. Elsevier Computers in Industry (2008).

[21] O. Serrate, T. Berger, D. Tabary and C. Kolski, Towards context-aware interactions between humans and a self-organized cybercar system. 27th European Annual Conference on Human Decision-Making and Manual Control, EAM'08 (2008).

[22] S. Shen and N. Wilson, Optimal integrated real-time disruption control model for rail transit systems. Computer-Aided Scheduling of Public Transport (2001) 335-364.

[23] M. Sugeno, Theory of fuzzy integrals and its applications. Ph.D. thesis, Tokyo Institute of Technology (1974)

[24] D. Trentesaux, T. Berger and Y. Sallez, Product and resource driven control of allocation and routing process in fms. MOSIM'08 (2008).

[25] R. Yager, The induced fuzzy integral aggregation operator. Int. J. Intelligent Syst. 17 (2002) 1049-1065. 